\title{
Etiology of Hydrops Fetalis at the Philippine General Hospital: A Retrospective Study
}

\author{
Michelle E. Abadingo and Maria Melanie Liberty B. Alcausin \\ Department of Pediatrics, College of Medicine and Philippine General Hospital, University of the Philippines Manila
}

\begin{abstract}
Objective. Hydrops fetalis (HF) is a diagnosis with significant morbidity and mortality. It is the objective of this study to identify common etiologic causes of HF in the Philippine General Hospital (PGH).

Methods. This is a retrospective review of hydrops fetalis cases delivered at the PGH from 2010 to 2014 . There was a total of 75 identified cases of hydrops fetalis. However, only 58 of these, or $77.33 \%$ have available medical charts for review.

Results. The median gestational age at birth was 31 (range 2140) weeks. There were 19 identified cases (32\%) of stillbirth. Of the 32 patients born alive, $30(93.75 \%)$ died in the immediate neonatal period. The etiologies of HF were identified in 15 cases, which included congenital anomalies $(n=4)$, cardiac anomalies $(n=3)$, infectious problems $(n=3)$, hematologic anomalies $(n=2)$, placental anomalies $(n=2)$ and congenital tumor $(n=1)$. Cases of congenital anomalies were only detected by congenital anomaly scan; no further work-ups were done. Fifteen (15) mothers presented with microcytic, hypochromic anemia on complete blood count $(\mathrm{CBC})$. The cause of HF was not confirmed in the remaining $43(74 \%)$ cases.
\end{abstract}

Conclusion. It is important to fully investigate the causes of HF to aid in the proper management and counseling. Further work-up must be done for mothers presenting with microcytic, hypochromic anemia. This is an important feature of alpha thalassemia. Partners of women suspected to have alpha thalassemia should also have a hematologic screening. The deletion of the four alpha genes will result to HB Bart's hydrops fetalis; a major cause of hydrops fetalis in Southeast Asian countries.

Key Words: Hydrops fetalis, etiology, fetal outcome

Paper presented as a poster at the 28th International Pediatrics Association Conference, August 17-22, 2016, Vancouver Convention Center, Vancouver, BC, Canada.

Corresponding author: Michelle E. Abadingo, MD

Department of Pediatrics

Philippine General Hospital

University of the Philippines Manila

Taft Avenue, Ermita, Manila 1000 Philippines

Telephone: +6325261710

Email: meabadingo@up.edu.ph

\section{Introduction}

Hydrops fetalis (HF) is defined as abnormal accumulation of serous fluid in two or more fetal compartments; such as skin, pleura, pericardium, peritoneum, placenta and amniotic fluid. There are two categories of hydrops fetalis: isoimmune (IHF) and nonisoimmune (NIHF). IHF results in erythroblastosis fetalis, where there is an increased rate of red blood cell (RBC) destruction due to transplacental passage of maternal antibody active against paternal RBC antigens of the infant; this entity is approximately 3 times more frequent in the Caucasian population. ${ }^{1}$ NIHF on the other hand develops in the absence of maternal antibody-fetal antigen mediated RBC destruction. ${ }^{2}$ At present, NIHF comprise $76-90 \%$ of all described HF cases. ${ }^{3,4}$

Several studies were done elucidating the possible causes of NIHF. While there are many identified causes of NIHF, increase in central venous pressure (CVP) is common in the pathophysiology of NIHF. This leads to accumulation of interstitial fluid, leading to hydrops fetalis. ${ }^{5}$ Cardiovascular anomalies was identified as a main etiologic factor for NIHF.3,6,7 However, certain populations may have increased propensity for NIHF due to particular medical conditions inherent in the population. An example would be HB Bart's hydrops fetalis syndrome wherein the absence of four alpha-globin genes would result into a severe form of anemia. If left untreated, this condition will result to death in the second or third trimester of gestation, or neonatal period. ${ }^{8-10}$ The most severe forms of alphathalassemia are restricted in the Southeast Asian region, which includes the Philippines and China. ${ }^{11}$ In Southeast Asia, alpha-thalassemia is the most common cause of fetal hydrops, accounting to $60 \%$ to $90 \%$ of cases. ${ }^{8,9}$ In a 2003 retrospective study done in MCU-Filemon D. Tantoco Medical Foundation (MCU-FDTMF) in the Philippines, thalassemias ranked third in the most common cause of hydrops fetalis. ${ }^{12}$

In a national database in the United States, 598 neonates of 253,651 discharges from neonatal ICUs (NICU), or $0.23 \%$ of discharges were identified with hydrops fetalis. Thirty-six percent of these patients died before hospital discharge. ${ }^{6}$ In a 2003 study done at the Philippine General Hospital (PGH), hydrops fetalis ranked $5^{\text {th }}$ in the most common congenital anomalies. ${ }^{13}$ In a more recent study done in the same institution, results showed that hydrops fetalis was one of 
the frequently encountered congenital anomalies detected by prenatal ultrasound. However, most of the hydropic fetuses did not have a complete work-up as to the possible etiology. ${ }^{14}$ Recent advances in prenatal ultrasound made it possible to identify hydrops fetalis early in gestation. However, despite this, fetuses with hydrops fetalis die antepartum or intrapartum with a mortality rate of 20$90 \% .{ }^{14,15}$ According to the 2012 health statistics of the Department of Health (DOH), conditions originating in the perinatal period (P00-P99) ranked tenth in the 10 leading causes of overall mortality. ${ }^{16}$ Hydrops fetalis is included in this cluster.

Hydrops fetalis is a diagnosis with significant morbidity and mortality. An epidemiologic profile of hydrops fetalis in the local population is needed to identify possible etiologic factors. This is particularly significant in countries like the Philippines where prenatal diagnosis is practiced in a limited way and termination of pregnancy is not allowed by law. ${ }^{17}$ A review of previous cases of NIHF would aid in appropriate management and treatment planning. The results can also be utilized in genetic counseling of families regarding management options available and risk of having further affected children. For ethnicities with increased risk for certain conditions such as alpha-thalassemia in Filipinos, the results can be used to strengthen and create policies regarding prenatal detection and patient education.

This retrospective study aimed to identify the etiologic profile of HF in the local population. The specific objectives of this study were: to identify delivered cases of HF at the PGH from 2010 to 2014; and to review the epidemiologic profile (maternal demographic characteristics, identification of possible maternal risk factors, antenatal diagnostic workups done, and birth outcomes) of hydrops fetalis cases.

\section{Methods}

\section{Description of the Study Setting}

The PGH is the largest tertiary hospital and referral center in the Philippines with a 1500-bed capacity. It has 14 clinical departments, namely: Anesthesiology, Emergency Medicine, Family Medicine, Internal Medicine, Neurosciences, Obstetrics and Gynecology, Ophthalmology, Orthopedics, Otorhinolaryngology, Pathology, Pediatrics, Psychiatry, Radiology and Surgery. The hospital also has the most number of subspecialty services. This includes the Section of Perinatology under the Department of Obstetrics and Gynecology; and Section of Neonatology under the Department of Pediatrics. As a tertiary referral center, it receives patient referrals needing specialized medical and surgical care from other institutions all over the country.

\section{Study Design and Population}

For the purpose of this study, hydrops fetalis is defined as excessive fluid accumulation within at least 2 extravascular compartments of the body. The study was reviewed and approved by the University of the Philippines Manila Research Ethics Board (UPMREB). Names and personal information were anonymized and kept confidential. The lists of hydrops fetalis deliveries were obtained from the Section of Perinatology, Department of Obstetrics and Gynecology, and Section of Neonatology, Department of Pediatrics. Medical charts of delivered cases at the PGH from January 1, 2010 to December 31, 2014 were retrieved from the Medical Records Section, Philippine General Hospital.

Maternal demographic data recorded were: initials, age, obstetric score, gestational age at time of diagnosis, number of prenatal visits, co-morbid conditions, previous history of hydrops fetalis and obstetric complications. Pertinent obstetric history and work-ups done during the course of the pregnancy were obtained.

Fetal data recorded were: dates of antenatal ultrasound, gestational aging at birth, pediatric aging, mode of delivery, birth weight, APGAR scores, fetal outcome and laboratory tests done.

\section{Data Analysis}

Clinical data collected were transferred to a passwordprotected Microsoft Excel (Microsoft Corporation, Redmond, WA, USA) spreadsheet file. Patients were assigned case codes that were used in recording data. Data were recorded and screened. The number of deliveries during the five-year study period was obtained from the records of the Section of Neonatology, Department of Pediatrics. The sample size for this study is 73 , using the formula $n=Z^{2} \times[p(1-p)] / e^{2}$, where $\mathrm{Z}=1.96, \mathrm{p}=0.05$ and $\mathrm{e}=0.05$.

\section{Results}

Table 1 shows the identified cases of hydrops fetalis from the Section of Perinatology, Department of Obstetrics and Gynecology. While the identified cases of hydrops fetalis met the computed sample size for the study $(n=75)$,

Table 1. Total number of deliveries and occurrence of patients with hydrops fetalis per 1,000 deliveries, 2010-2014

\begin{tabular}{cccc}
\hline Year & $\begin{array}{c}\text { Total number } \\
\text { of deliveries }\end{array}$ & $\begin{array}{c}\text { Total number of patients } \\
\text { with hydrops fetalis }\end{array}$ & $\begin{array}{c}\text { Cases reviewed } \\
\text { n (\%) }\end{array}$ \\
\hline 2010 & 5852 & 18 & $6(33.33)$ \\
2011 & 5710 & 16 & $16(100)$ \\
2012 & 5120 & 12 & $7(58.33)$ \\
2013 & 4692 & 11 & $11(100)$ \\
2014 & 5340 & 18 & $18(100)$ \\
TOTAL & $\mathbf{2 6 , 7 1 4}$ & $\mathbf{7 5}$ & $\mathbf{5 8}(\mathbf{7 7 . 3 3 )}$ \\
\hline
\end{tabular}


only 58 (77.33\%) have medical records available for review. During the study period, there were 26,714 live births giving an incidence for HF of 2.77/1000 live births.

Mean maternal age is 28 years, and median age of gestation is 31. Majority of those in the study were multigravid (91.53\%); four (4) had a previous history of hydrops fetalis delivery. The gestational age when hydrops fetalis was first detected was not indicated in most cases. One of the women who had a previous hydrops fetalis delivery had her deliveries in this institution. For the two women who had twin pregnancies, both delivered neonates who underwent twin-to-twin transfusion syndrome (Table 2).

Table 2. Maternal demographics and risk factors for hydrops fetalis pregnancy

\begin{tabular}{lc}
\hline VARIABLE & $\mathbf{n} \mathbf{( \% )}$ \\
\hline Maternal age (years) & $28.5 \pm 6.08$ \\
Age of gestation on delivery & $31^{*}$ \\
Gravidity & $7(12.07)$ \\
Primigravid & $51(87.93)$ \\
Multigravid & \\
Number of pregnancy & $56(96.55)$ \\
$\quad$ Singleton pregnancy & $2(3.45)$ \\
$\quad$ Twin pregnancy & \\
Maternal risk factors for hydrops fetalis & $4(6.90)$ \\
Previous hydrops fetalis pregnancy & $15(25.96)$ \\
Anemia & 2 \\
$\quad$ Alpha thalassemia & $3(5.17)$ \\
Infection & 1 \\
$\quad$ Toxoplasmosis & 2 \\
$\quad$ Syphilis & \\
Maternal complication secondary to HF & $3(5.17)$ \\
$\quad$ Mirror syndrome &
\end{tabular}

All mothers had complete blood count (CBC) done prior to delivery. Of the 58 charts reviewed, 15 mothers presented with microcytic, hypochromic anemia. For this study, microcytic anemia was defined as having mean corpuscular volume $(\mathrm{MCV})$ of less than 83 and mean corpuscular hemoglobin $(\mathrm{MCH})$ of less than 27. Of the 15 women, one was identified to have alpha thalassemia trait. Another woman was confirmed to have alpha thalassemia. For the rest of the women with microcytic, hypochromic anemia, no further investigations were done to probe on the possible etiology of the anemia. Paternal CBC results were not documented in the newborn charts.

Infection is identified as an etiologic factor for hydrops fetalis. Identified etiologic agents in the study include toxoplasmosis and syphilis. In this study, one woman had an active toxoplasma infection (IgM reactive); while two other women had syphilis infection.

Mirror syndrome, or Ballantine syndrome, is the association of fetal and placental hydrops with maternal preeclampsia. This was seen in three mothers in this study.

Relevant neonatal demographics are presented in Table 3. Most deliveries had Pediatric Aging (PA) of 30, with PA range of 23 to 40. Majority were delivered by cesarean section. Indications for cesarean section include deteriorating fetal status, multiple gestation, maternal Mirror Syndrome and identified fetal multiple anomalies on congenital anomaly scan (CAS). The male-to-female ratio is 1:1.3. The median APGAR score at one minute is 2; while the median APGAR score at five minutes is 4 . While $32(54.24 \%)$ neonates were delivered alive, majority $(93.75 \%)$ died in the immediate neonatal period. Two of the deliveries born alive had documented advanced directives of do not intubate (DNI) and do not resuscitate (DNR). No documentation was done on the resuscitation and management after delivery. There were no fetal autopsies documented.

Table 3. Neonatal demographics

\begin{tabular}{lc}
\hline & $\mathbf{n}(\mathbf{\%})$ \\
\hline Pediatric aging & $30(23-40)^{*}$ \\
Mode of delivery & $10(17.24)$ \\
Normal vaginal delivery & $5(8.62)$ \\
Assisted vaginal delivery & $39(67.24)$ \\
Cesarean section & $4(6.90)$ \\
Unspecified & $2,300(500-4,300)^{*}$ \\
Median birth weight & \\
Sex & $23(36.66)$ \\
Male & $29(50.00)$ \\
Female & $1(1.72)$ \\
Ambiguous & $5(8.62)$ \\
Unspecified & \\
APGAR score & $2(0-9)^{*}$ \\
1 minute & $4(0-9)^{*}$ \\
5 minutes & \\
Fetal outcome & $19(32.76)$ \\
Stillbirth & $32(55.17)$ \\
Alive & $30(93.75)$ \\
Died in the neonatal period & $7(12.07)$ \\
Undetermined & \\
\hline
\end{tabular}

${ }^{*}$ Data presented as range

Coombs test was done for $16(27 \%)$ cases. For the 16 cases, all had negative direct and indirect Coombs. Causes of hydrops fetalis were identified in 14 cases (Table 4).

Table 4. Determined causes of Hydrops Fetalis

\begin{tabular}{lc}
\hline & $\mathbf{n ~ ( \% )}$ \\
\hline Congenital anomaly & $\mathbf{4}(\mathbf{7})$ \\
\hline Skeletal dysplasia & $2(3)$ \\
Multiple congenital anomaly (hydrocephalus, & \\
cardiomegaly, pelvocaliectasia, distended bladder) & $1(2)$ \\
Chromosomal Anomaly (Trisomy 21) & $1(2)$ \\
Cardiac & $3(5)$ \\
Fetal heart block & $1(2)$ \\
Fetal cardiomegaly & $1(2)$ \\
Coarctation of aorta & $1(2)$ \\
$\underline{\text { Infectious }}$ & $3(5)$ \\
Toxoplasmosis & $1(2)$ \\
Syphilis & $2(3)$ \\
Placental abnormality & $\mathbf{2}(3)$ \\
Twin-to-twin transfusion syndrome & $2(3)$ \\
$\underline{\text { Hematologic }}$ & $2(3)$ \\
C-thalassemia & $2(3)$ \\
$\frac{\text { Congenital tumor }}{\text { Cervical teratoma }}$ & $\mathbf{1}(2)$ \\
$\frac{\text { Totetermined }}{\text { TOTAL }}$ & $1(2)$ \\
\hline
\end{tabular}


For this study, the probable causes of HF were classified as infectious, hematologic, placental abnormality, cardiac, and congenital tumor. Cause of hydrops fetalis was undetermined for majority of the cases. Only 14 (24\%) women were screened for intrauterine infections. However, most were only screened for syphilis and TORCH (Toxoplasma, Rubella, Cytomegalovirus, Herpes) infection. Hemoglobin electrophoresis was only done in two of the 15 patients presenting with microcytic, hypochromic anemia on CBC. Fetal cardiac anomalies were identified by fetal 2Dechocardiography. The case of cervical teratoma was identified by fetal Congenital Anomaly Scan (CAS).

There were 4 cases of multiple congenital anomalies (MCA) identified by CAS (Table 4). Two were cases of skeletal dysplasias while one is a possible case of Trisomy 21. However, chromosomal analysis results and other investigations were not documented for these cases.

\section{Discussion}

Hydrops fetalis can be categorized as isoimmune and non-isoimmune. Coombs test can be done to differentiate between isoimmune and non-isoimmune HF. ${ }^{18}$ It is an important initial tool in directing the work-up for HF. In this study, Coombs test was only done for 16 (27\%) cases. For the 16 cases, the probable cause of HF is non-isoimmune since all were negative for both direct and indirect Coombs.

Neonatal demographics of the study show that neonates were delivered preterm (median PA 30) with a median APGAR score at one minute of two (2) and four (4) at five minutes. In a previous study by Abrams et al., ${ }^{6}$ infants who died were more likely to be premature and sicker (i.e. lower 5-minute APGAR scores). In the same study, patients discharged had a median PA of 34 and APGAR score of $>3$ in the $1^{\text {st }}$ and $5^{\text {th }}$ minute. In this study, the relatively low pediatric aging and APGAR scores may be attributed to lack of prenatal work-ups done for both the mother and the fetus. There was no available data on the number of prenatal consults that the mothers had. As a tertiary referral center, PGH receives imminent deliveries from other institutions.

Ideally, confirmatory tests are done upon the antenatal diagnosis of hydrops fetalis by ultrasound. This include congenital anomaly scan, fetal echocardiography and Doppler studies. Further investigation is done to the mother and fetus. Maternal diagnostic work-ups include CBC, blood group and antibodies, hemoglobin electrophoresis, TORCH screen, VDRL, autoantibody screen for cases of congenital heart blocks and oral glucose test. Additional fetal work up includes fetal blood sampling. Prenatally, amniotic fluid sampling, fetal karyotyping and genetic microarray testing are also done. Autopsy is recommended for all neonatal and fetal deaths. ${ }^{18,19}$

Five percent of the reviewed cases had cardiac anomalies. In this study, cardiac anomalies were identified by fetal 2D-echocardiography. In a previous retrospective study, congenital heart disease and cardiac arrhythmias were the most common causes of hydrops fetalis. ${ }^{6}$ Structural cardiac anomalies lead to high right atrial pressure or volume overload and right-sided heart congestion. This in turn leads to increased central venous pressure and heart failure; or obstruction of venous and arterial blood flow which would lead to edema. ${ }^{3,5}$

Complete blood count (CBC) is a basic laboratory workup noted in the cases reviewed. Microcytic, hypochromic anemia is commonly seen in alpha-thalassemia. In our study, 15 mothers presented with microcytic, hypochromic anemia. Alpha thalassemia is frequent in Mediterranean and Southeast Asian countries, which includes the Philippines. ${ }^{10}$ It is important to also screen the partners of women suspected to have alpha thalassemia. In this study, paternal $\mathrm{CBC}$ was not documented in the charts reviewed.

Deletion of the four alpha-globin chains would result into $\mathrm{Hb}$ Bart's hydrops fetalis syndrome, the fatal form of the alpha thalassemia syndromes. ${ }^{9-11}$ The deletion of all the alpha-genes is inherited from both parents; with both parents having alpha thalassemia. The absence of the fourglobin chains would result into anemia and hypoxia. There would then be an increase in cardiac output which will progress to cardiac failure. Increased central venous pressure would result from cardiac failure; this would then result to increase in interstitial fluid and decreased lymph flow. The accumulation of interstitial fluid would explain the presence of edema in hydrops fetalis. 3,5

Aside from microcytosis, carriers are detected on the basis of normal serum ferritin. A clinical practice guidelines in the management of non-immune hydrops fetalis recommends a routine hemoglobin electrophoresis screening in ethnicities with high frequencies of alpha thalassemia. ${ }^{19}$ In a previous study done in another Southeast Asian country, Thailand, $36.58 \%$ of the cases of HF were due to Hb Bart's hydrops fetalis. ${ }^{20}$ In another study done in Singapore, HF secondary to homozygous alpha-thalassemia is relatively low at $7 \%$ due to antenatal thalassemia screening. ${ }^{7}$ In the study by Fucharoen and Winichagon in 2007, wherein the prevention and control of thalassemia were discussed, it was identified that prenatal diagnosis and genetic counselling are done ideally for high-risk couples. ${ }^{21}$

Hydrops fetalis secondary to intrauterine infections (IUI) were also observed in the reviewed cases. Work-ups were done for the TORCHES (Toxoplasma, Rubella, Cytomegalovirus, Herpes, Syphilis) infection. Test for Parvovirus B19 and varicella should also be included in the work-up for hydrops fetalis. ${ }^{19}$ Intrauterine infections affect the fetal liver, which is the site of erythrocyte production during early development. ${ }^{19,22}$ This then leads to fetal anemia, which would increase cardiac output. This results into congestive heart failure and increased central venous pressure. $^{5}$ 
There were four identified cases of hydrops fetalis with concomitant congenital anomalies. One is a suspected case of Trisomy 21 while two were cases of skeletal dysplasias. Skeletal dysplasias involve deformation of the thoracic skeleton; causing constriction of the thoracic viscera. ${ }^{3}$ In the study done by Abrams et al. in 2007, mortality rates for HF were highest among neonates with associated congenital anomalies. $^{6}$ For the patient with multiple congenital anomalies, hydrops fetalis may be secondary to a cardiac anomaly presenting as fetal cardiomegaly.

The results of this retrospective study show the importance of knowing the etiology of hydrops fetalis. The findings can be used to counsel pregnant women about the investigation, the prognosis of the disease, as well as the optimal modalities for management. ${ }^{20}$ Genetic counseling is particularly important for cases of hydrops fetalis that are hereditary; such as alpha thalassemia. ${ }^{21}$ If diagnosed prenatally, in-utero interventional measures may be done depending on the etiology of hydrops fetalis: fetal intravascular transfusion for fetal anemia, direct fetal drug therapy for fetal arrhythmia and pleuro-amniotic shunt for pleural effusion. ${ }^{18}$

As a retrospective study, this paper has many limitations. Data available in the charts may be incomplete. Not all charts were available for review. The Sections of Neonatology and Perinatology do not have duplicate copies of the medical charts of HF cases. Currently, there is no available database for $\mathrm{HF}$ cases in PGH. Information on severity of prenatal presentation and intervention were also not available. These would had been important in determining the previous work-ups done for HF.

\section{Conclusion}

It is important to determine the etiology of $\mathrm{HF}$ to explain its overlying pathophysiology. In this study, majority of the cause of $\mathrm{HF}$ is undetermined. Majority $(93.75 \%)$ of those born alive died in the immediate neonatal period. Knowing the etiology of this condition would aid in treatment planning and appropriate parental genetic counseling. Furthermore, knowing the common causes of $\mathrm{HF}$ in our population would also aid in designing the appropriate framework for the work-up of cases of hydrops fetalis. For suspected hereditary causes of hydrops fetalis, such as alpha thalassemia, paternal work-ups should also be done to support the diagnosis.

\section{Recommendations}

A local database of hydrops fetalis is recommended for cases to be properly documented. Further studies are also recommended to look into the etiologic factors of hydrops fetalis. In a low-resources setting, it is important for laboratory work-ups to be directed, depending on the etiology of hydrops fetalis. A local systematic guideline for the work-up of hydrops fetalis is recommended to ensure a complete search of its etiology. An ongoing study in PGH investigates the etiology of hydrops fetalis, prospectively. In the case of the Philippines where alpha-thalassemia is more frequent, prenatal screening and genetic counseling should be done. The results of this study, along with a more extensive prospective study, can be used to draft health policies regarding prenatal screening of genetic disorders.

\section{Statement of Authorship}

All authors have approved the final version submitted.

\section{Author Disclosure}

All the authors declared no conflicts of interest.

\section{Funding Source}

None.

\section{References}

1. Maheshwari A,Carlo WA. Hemolytic Disease of the Newborn. In: Kliegman RM, Stanton BF, St Geme III JW, et al., eds. Nelson Textbook of Pediatrics, $20^{\text {th }}$ ed. Philadelphia: Elsevier Saunders; 2016. pp. 883-887.

2. Chen JY. Etiology and outcome of Hydrops Fetalis. Pediatr Neonatol. 2014; 55(2):77.

3. Bellini C, Hennekam RC, Fulcheri E, et al. Etiology of nonimmune hydrops fetalis: A systematic review. Am J Med Genet A. 2009; 149A(5):844-51.

4. Yurdakok M. Non-immune hydrops fetalis. J Pediatr Neonat Individual Med. 2014; 3(2): e030214.

5. Bellini C, Hennekam RC. Non-immune Hydrops Fetalis: a short review of etiology and pathophysiology. Am J Med Genet A. 2012; 158A(3):597605.

6. Abrams ME, Meredith KS, Kinnard P, Clark RH. Hydrops Fetalis: a retrospective review of cases to a large national database and identification of risk factors associated with death. Pediatrics. 2007; 120(1):84-9.

7. Ng ZM, Seet MJ, Erng MN, Buendia F, Chang AS, Sriram B. Nonimmune hydrops fetalis in a children's hospital: a six-year series. Singapore Med J. 2013; 54(9):487-90.

8. Chui DH, Waye JS. Hydrops fetalis caused by $\alpha$ Thalassemia: an emerging health care problem. Blood. 1998; 91(7):2213-22.

9. Lorey F, Charoenkwan $\mathrm{P}$, Witkowska $\mathrm{E}$, et al. $\mathrm{Hb} \mathrm{H}$ hydrops foetalis syndrome: a case report and review of literature. Br J Haematol. 2001; 115(1):72-8.

10. Harteveld CL, Higgs DR. Alpha thalassemia. Orphanet J Rare Dis. 2010; 5:13.

11. Singer ST. Variable clinical phenotypes of $\alpha$-Thalassemia Syndromes. Scientific World Journal. 2009; 9:615-25.

12. Gamutan KGC, F Lucero-Tan. Non-immune hydrops fetalis: A review of 5 years' experience (Abstract). Philipp Scient J. 2003; 36(1):15-24.

13. Padilla CD, Cutiongco EM, Sia JM. Birth Defects ascertainment in the Philippines. Southeast Asian J Trop Med Public Health. 2003; 34 Suppl 3:239-43.

14. Abarquez CG, Alcausin MMB, David-Padilla C, Cutiongco-Dela Paz EM. A five-year review of cases referred to the Genetics Clinic, PGH for findings of congenital anomalies on prenatal ultrasound. Acta Med Philipp. 2009; 43(1):22-8.

15. Liu CA, Huang HC, Chou YY. Retrospective analysis of 17 liveborn neonates with Hydrops Fetalis. Chang Gung Med J. 2002; 25(12):826-31. 
16. Department of Health. The 2012 Philippine Health Statistics [Online]. 2012 [cited 2016 Sep]. Available from http://www.doh.gov.ph/sites/ default/files/publications/PHS2012.pdf.

17. David-Padilla C, Dela Paz EMC. Genetic services and testing in the Philippines. J Community Genet. 2013; 4(3):399-411.

18. Anandakumar C, Biswas A, Wong YC, et al. Management of nonimmune hydrops: 8 years' experience. Ultrasound Obstet Gynecol. 1996; 8(3):196-200.

19. Desilets V, Audibert F, Society of Obstetricians and Gynecologists of Canada. Investigation and Management of Non-immune Fetal Hydrops. J Obstet Gynaecol Can. 2013; 35(10):923-38.
20. Ratanasiri T, Komwilaisak R, Sittivech A, Kleebkeaw P, Seejorn K Incidence, causes and pregnancy outcomes of Hydrops Fetalis at Srinagarind Hospital, 1996-2005: A 10-year review. J Med Assoc Thai. 2009; 92(5):594-9.

21. Fucharoen S, Winichagoon P. Prevention and control of thalassemia in Asia. Asian Biomedicine. 2007; 1 (1):1-6.

22. Figueiredo CA, de Oliveira MI, Afonso AMS, et al. Detection of human Parvovirus B19 in cases of hydrops fetalis in Sao Paulo, Brazil. J Bras Patol Med Lab. 2008; 44(6):423-7.

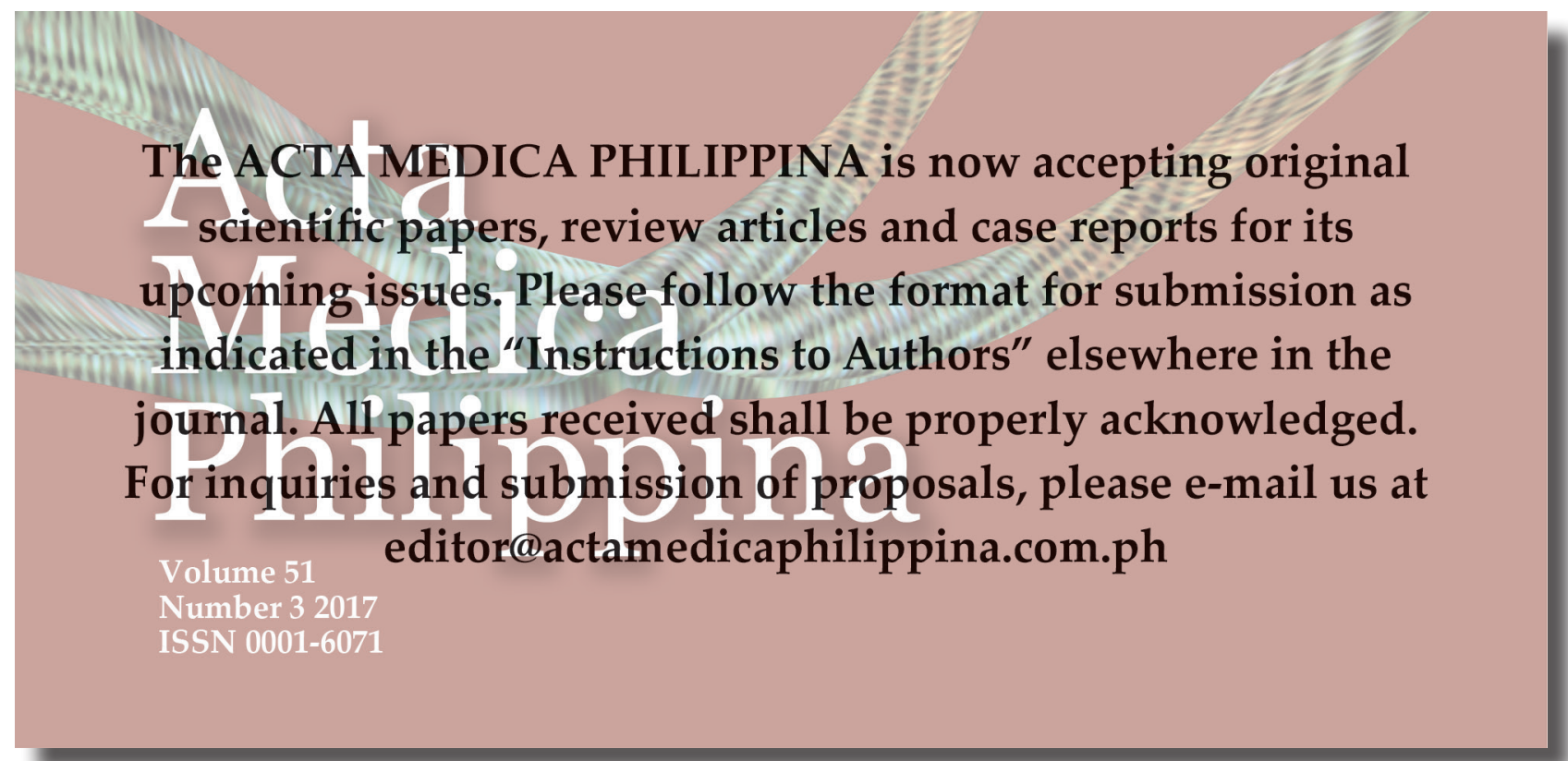

